Pacific Journal of Mathematics

ON THE STRUCTURE OF ALMOST PERIODIC 


\title{
ON THE STRUCTURE OF ALMOST PERIODIC TRANSFORMATION GROUPS
}

\author{
HSIN CHU
}

\begin{abstract}
In this note, among other things, we shall establish the following result:

Theorem. Let $(H, X, \pi)$ be an effective transformation group, where $X$ is a compact 3-dim, connected manifold, $H$ is a connected locally compact but non-compact topological group and $H$ acts on $X$ almost periodically. Then $H$ must be a Lie group.
\end{abstract}

Moreover, If $H$ is abelian, $H$ must be continuously isomorphic into and dense in a torus group of either dimension two or dimension three. In the case of a three-dimensional torus, $X$ must be a minimal set and itself homeomorphic to a three-dimensional torus. The action of $H$ is the usual group multiplication. In the case of a twodimensional torus, $X$ must not be a minimal set and it must be homeomorphic to one of the following spaces:

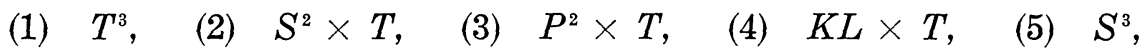

(6) $\left(\mathrm{Cl}\left(E_{2}\right) \times T\right) \cup(\mathrm{Cl}(M B) \times T)$, intersecting in a circle which, in the right side, is an infinite cycle and, in the left side, a cycle homologous to zero,

where (3), (4), and (6) are non-orientable, and $T^{m}$ is the $m$-dim torus, $S^{m}$ the $m$-sphere, $P^{m}$ the real projective $m$-space, $K L$ the Klein bottle, $M B$ the open Moebius strip and $E_{m}$ the open $m$-cell.

If $H$ is not abelian, $H$ must be continuously isomorphic into and dense in one of the following groups:
$\left(b_{1}\right) S O(3)$,
$\left(b_{2}\right) S U(2)$,
$\left(b_{3}\right) S O(3) \times T$,
$\left(b_{4}\right) S O(3) \times S U(2)$,
$\left(b_{5}\right) S O(3) \times S O(3)$,

where $S O(3)$ is the special orthogonal group of order $3, S U(2)$ the special unitary group of order 2 and $T$ the circle group. In the case of the last four groups, $X$ must be a minimal set and homogeneous space, but not a quotient group, of each respective group. In the case of the first group, $X$ must not be a minimal set and must be homeomorphic to one of the following spaces:
(1) $S^{2} \times T$,
(2) $P^{2} \times T$,
(3) $S^{3}$,
(4) $P^{3}$
(5) $\quad\left(P^{3} \backslash E_{3}\right) \cup\left(P^{3} \backslash E_{3}\right)$ intersecting in $\mathrm{Cl}\left(E_{3}\right) \backslash E_{3}$
(6) $S^{2} \times I$, identifying $(x, 0)$ with $(-x, 1)$.

where (2) and (6) are non-orientable manifolds and $I$ is the closed unit interval.

The main tool of the proof of this theorem is to associate the 
given transformation group with a compact transformation group and apply a partially affirmative answer to the Hilbert-Montgomery conjecture. Consequently, in general, we are unable to extend the result beyond dimension three. However, we do extend this result to the case of minimal sets.

2. Definition and proof. We adopt the following standard definition (see [3] and [4]).

Definition. Let $(H, Y, \pi)$ be a transformation group, where $X$ is compact Hausdorff. This transformation group is called almost periodic if $\beta$ is an index of uniformity in $X$ implies there exists a syndetic subset $A$ of $H$ such that for $x \in X$, we have $\pi(A, x) \subset \beta(x)$. A subset $A$ in $H$ is called syndetic if there is a compact subset $K$ in $H$ such that $A \cdot K=H$. This transformation group is called a minimal set if the smallest invariant closed set, under $H$, in $X$ is $X$ itself.

Proof of the theorem. Since $X$ is compact Hausdorff, it is known (see [5]) that $H$ acts on $X$ almost periodically if and only if the action of $H$ on $X$ is uniformly equicontinuous. Consider $f . H \rightarrow X^{X}$ for each $t \in H$, $f(t)=\{\pi(t, x) \mid x \in X\}$, where $X^{X}$ is the set of all maps of $X$ into $X$ with its usual cartesian product topology, which is compact Hausdorff. Let $G=\mathrm{Cl}(f(H))$. Since the action of $H$ on $X$ is uniformly equicontinous it is easy to see that $G$, as a group of homeomorphisms on $X$, is a compact topological group. Define $G \times X \stackrel{\pi^{\prime}}{\rightarrow} X$ by $\pi^{\prime}(g, x)=g(x)$, where $g \in G$ and $x \in X$. It is not hard to verify that $\left(G, X, \pi^{\prime}\right)$ is a transformation group and the following diagram is commutative:

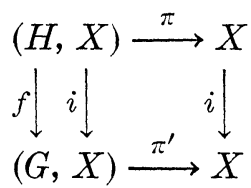

where $i$ is the identity map. The group $H$ acts on $X$ effectively; so does $G$. The group $H$ is connected; so is $G$. It is known that if a compact, connected group acts on a compact three-dimensional manifold effectively, the group must be a Lie group (e.g., see [8]). Since $H$ is locally compact and $F$ is one-to-one into the Lie group $G, H$ must be a Lie group. It is well known that if the maximal dimension of any orbit, under $G$, is $k$, then the dimension of $G$ is less than or equal to $1 / 2 k(k+1)$. If $G$ is abelian, then $G$ is a torus. Suppose $k=1$, then $\operatorname{dim} . G=1$ and $G$ is the circle group. Since $H$ is connected, but not compact and $f(H)$ is a nontrivial connected 
subgroup of $G$, we have $f(H)=G$ and $f$ is onto and open. It follows that $f$ is a topological isomorphism and $H$ itself is a circle group. This is a contradiction to the fact that $H$ is not compact. Hence, $k \neq 1$. If $k=2$, then $2 \leqq \operatorname{dim} . G \leqq 3$ and $G$ is a torus of dimension either 2 or 3 . Suppose $\operatorname{dim} . G=3$. Then, from the fact that the maximal dimension of any orbit under $G$ is 2 , there exists $x_{0} \leqq X$ such that its isotropy subgroup $G_{x_{0}}$ is of dimension one. Thus $G_{x_{0}}$ contains a circle group and we may write $G_{x_{0}}=T \times D$, where $T$ is the circle group and $D$ is a finite group. For $x \in X$, we denote by $m(x)$ the number of components in the isotropy subgroup $G_{x}$ of $x$. Let $X_{2}=\{x \mid x \in X$, dim. $G(x)=2\}$. Let $s=\min . m(x), x \in X_{2}$. It is known that the set $X_{2, s}=\left\{x \mid x \in X_{2}, m(x)=s\right\}$, which is called the union of all principal orbits, is dense in $X$ (see [5]). Since $G$ is connected, it is known that $X_{2, s}$ is connected (see [5]). It is also known that, for each $x \in X$, there exists a neighborhood $U_{x}$ of $x$ such that for each $y \in U_{x}, G_{y}$ is conjugate to a subgroup of $G_{x}$ (e.g., see [6]). Since $G$ is abelian, $G_{y}$ must be a subgroup of $G_{x}$. It follows that $X_{2, s}$ is open and every $G_{x}$ for $x \in X_{2, s}$ contains a circle group, $T^{\prime}$. Since $\bar{X}_{2, s}=X$, we can conclude that for each $x \in X, G_{x}$ contains the same circle group $T^{\prime}$. This gives a contradiction to the fact that $G$ acts on $X$ effectively. Hence, if $k=2$, the group $G$ must be a two-dimensional torus. In this case, $G$ can not act on $X$ transitively; it follows that $X$ is not minimal under $H$. If $k=3$, then $3 \leqq \operatorname{dim} . G \leqq 6$ and $G$ must act on $X$ transitively. Suppose $\operatorname{dim} . G>3$, then for each $x \in X$, dim. $G_{x} \geqq 1$. From the fact that $G$ is abelian, it follows that $G$ can not act on $X$ effectively. Hence, if $k=3$, the group $G$ must be a three-dimensional torus and for each $x \in X, G_{x}$ is trivial. Consequently, we can introduce a torus group structure on $X$, so that the action of the group $G$ on $X$ is just the group multiplication. It follows that the action of $H$ on $X$ is the same group multiplication. In the case that $k=2$, the group $G$ is a two-dimensional torus and the space $X$ is a three-dimensional compact connected manifold. It is known (e.g., see theorem 7, [7]) that $X$ must be homeomorphic to one of the following spaces:
(1) $T^{3}$,
(2) $S^{2} \times T$
(3) $P^{2} \times T$,
(4) $K L \times T$,
(5) $S^{3}$,

(6) $\left(\mathrm{Cl}\left(E_{2}\right) \times T\right) \cup(\mathrm{Cl}(M B) \times T)$, intersecting in a circle which, in the right side, is an infinite cycle and, in the left side, a cycle homologous to zero.

Now, consider the case that $G$ is not abelian. If $k=1$, then $\operatorname{dim} . G=1$. This can not happen because there are no one-dimensional compact connected, non-abelian, Lie groups. If $k=2$, then $2 \leqq$ $\operatorname{dim} . G \leqq 3$. There are only two three-dimensional compact connected, non-abelian, Lie groups, that is $S U(2)$ and $S O(3)$. There are no 
two-dimensional compact connected, non-abelian, Lie groups. Suppose $G=S U(2)$. If follows that for each $x \in X$, dim. $G_{x} \geqq 1$. It is known that any nonnormal subgroup of $S U(2)$ must contain its center $C$, where $C=\left\{\left(\begin{array}{ll}1 & 0 \\ 0 & 1\end{array}\right),\left(\begin{array}{rr}-1 & 0 \\ 0 & -1\end{array}\right)\right\}$. There are no other discrete, nontrivial, normal subgroup other than $C$. Since $S U(2)$ is simple, every isotropy subgroup $G_{x}$ is nonnormal. It follows that $G_{x} \supset C$ for every $x \in X$ and $G$ can not act on $X$ effectively. A contradiction! Hence, if $k=2, G$ must be $S O(3)$. Since $k=2, G$ can not act on $X$ transitively; it follows that $X$ is not minimal under $H$. In this case, it is known (e.g., see Theorem 7, [7]) that $X$ must be homeomorphic to one of the following spaces:
(1) $S^{2} \times T$,
(2) $P^{2} \times T$,
(3) $S^{3}$,
(4) $P^{3}$,
(5) $\quad\left(P^{3} \backslash E_{3}\right) \cup\left(P^{3} \backslash E_{3}\right)$ intersecting in $\mathrm{Cl}\left(E_{3}\right) \backslash E_{3}$
(6) $S^{2} \times I$, identifying $(x, 0)$ with $(-x, 1)$.

If $k=3$, then $3 \leqq \operatorname{dim} . G \leqq 6$ and $G$ must act on $X$ transitively. Consequently, $X$ is a minimal set under $H$. If $\operatorname{dim} . G=3$, then $S U(2)$ and $S O(3)$ are the only two non-abelian, compact, connected, Lie groups. It is possible, for example, that $S U(2)$ and $S O(3)$ can act on themselves with their group multiplications. If $\operatorname{dim} . G=4$, then $\operatorname{dim} G_{x} \geqq 1$ for every $x \in X$ and there is $x \in X$ such that $\operatorname{dim} G_{x}=$ 1. The only possible four-dimensional, non-abelian, compact, connected, Lie groups are $S U(2) \times T$ and its quotient groups by factoring a discrete normal subgroup. Since every nonnormal subgroup of $S U(2)$ contains its center $C$, it is not hard to see that every nonnormal subgroup of $S U(2) \times T$ contains a nontrivial central subgroup of $S U(2) \times T$ whose projection on $S U(2)$ is $C$. From the fact that $\operatorname{dim} . G_{x} \geqq 1$ for every $x \in X, G_{x}$ must contain a nontrivial normal subgroup. This is impossible because $\left(G, X, \pi^{\prime}\right)$ is a transitive and effective transformation group. Thus it is not hard to see now that the only possible case left is the group $(S U(2) / C) \times T$, which is isomorphic to $S O(3) \times T$. Indeed, this is possible; for example, let

$$
L=\left\{\left(\begin{array}{ccc}
\cos \theta & \sin \theta & 0 \\
-\sin \theta & \cos \theta & 0 \\
0 & 0 & 1
\end{array}\right) \mid-\pi \leqq \theta \leqq \pi\right\}
$$

be a circle group in $S O(3)$, and choose $X=(S O(3) / L) \times T$. Let $S O(3) \times T$ act on $X$ by the action induced by the group multiplication in $S O(3) \times T$. It is easy to verify that for every $x \in X, G_{x}$ is conjugate to $L \times\{0\}$ and $S O(3) \times T$ acts on $X$ effectively. If $\operatorname{dim} . G=5$, then $\operatorname{dim} . G_{x} \geqq 2$ for every $x \in X$ and there is $x \in X$, such that $\operatorname{dim} . G_{x}=2$. The only possible five-dimensional non-abelian, compact, connected, Lie groups are $S U(2) \times T^{2}$ and its quotient groups 
by factoring a discrete normal subgroup. Choose a $y \in X$ such that $\operatorname{dim} . G_{y}=2$. On one hand, since $\left(G, X, \pi^{\prime}\right)$ is transitive and effective, the group $G_{y}$ can not contain a nontrivial normal subgroup. On the other hand, from the fact that $\operatorname{dim} . G_{y}=2$, it is not hard to see that $G_{y}$ must contain a circle group which is contained in the center. A contradiction! Hence it is impossible that $\operatorname{dim} . G=5$ and $k=3$. If $\operatorname{dim} . G=6$, then $\operatorname{dim} . G_{x} \geqq 3$ for every $x \in X$ and there is $x \in X$, such that dim. $G_{x}=3$. The only possible six-dimensional, non-abelian, compact, connected, Lie groups are $S U(2) \times T^{3}, S U(2) \times S U(2)$ and their quotinent groups by factoring a discrete normal subgroup. If $G=S U(2) \times T^{3}$ or its quotient groups by factoring a discrete normal subgroup, then, from the fact that there is $y \in X$ such that $\operatorname{dim} . G_{y}=$ $3, G_{y}$ must contain a nontrivial normal subgroup. This gives a contradiction to the fact that $\left(G, X, \pi^{\prime}\right)$ is transitive and effective. The group $G$ also can not be $S U(2) \times S U(2)$. Otherwise any nonnormal subgroup must contain a nontrivial central subgroup which is contained in $C \times C$; then $G$ can not act on $X$ effectively. Since every discrete normal subgroup of $S U(2) \times S U(2)$ is in the center, $C \times C$, there are four possibilities left: (1) $S U(2) \times S U(2) / C \times C$, which is isomorphic to $S O(3) \times S O(3),(2) S U(2) \times S U(2) /\{e\} \times C$, which is isomorphic to $S U(2) \times S U(3)$, (3) $S U(2) \times S U(2) / C \times\{e\}$, which is isomorphic to $S O(3) \times S U(2)$, (4) $S U(2) \times S U(2) / C^{\prime}$, where

$$
C^{\prime}=\left\{\left(\left(\begin{array}{ll}
1 & 0 \\
0 & 1
\end{array}\right),\left(\begin{array}{ll}
1 & 0 \\
0 & 1
\end{array}\right)\right),\left(\left(\begin{array}{rr}
-1 & 0 \\
0 & -1
\end{array}\right),\left(\begin{array}{rr}
-1 & 0 \\
0 & -1
\end{array}\right)\right)\right\} \text {. }
$$

It is easy to see that the groups (2), (3) and (4) are isomorphic. It is easy to construct homogeneous spaces so that the action of these groups on the spaces satisfies the given conditions. The theorem is proved.

Since the proof of this theorem depends on a partially affirmative answer to the Hilbert-Montgomery conjecture on effective compact transformation on a manifold, we are unable to extend this result beyond dimension three. However, using the same method and a known result, which says that an effective and transitive compact transformation group acting on a connected finite-dimensional manifold is a Lie group (e.g., see [6]), we can have the following result:

Corollary. Let $(H, X, \pi)$ be an effective almost periodic transformation group, where $X$ is a compact, connected, finite dimensional manifold and $X$ is a minimal set under $H$. Then $X$ must be a homogeneous space of a compact, connected Lie group and the action of each element in $H$ on $X$ must be analytic. Furthermore, if $H$ is locally compact, then $H$ must be a Lie group. 


\section{REFERENCES}

1. G. D. Birkhoff, Dynamical systems, Amer. Math. Soc. Colloq. Pub., 9 (1927).

2. Hsin Chu, A remark on a conjecture of Seifert, to appear Topology.

3. W. H. Gottschalk and G. A. Hedlund, Topological dynamics, Amer. Math. Soc. Colloq. Publication vol. 36 (1955).

4. W. H. Gottschalk, Characterization of almost periodic transformation groups, Proc. Amer. Math. Soc., 7 (1956), 709-712.

5. D. Montgomery and C. T. Yang, Orbits of highest dimension, Trans. Amer. Math. Soc., 87 (1958), 284-293.

6. D. Montgomery and L. Zippin, Topological transformation groups, Interscience Publisher, New York, 1955.

7. P. S. Moster, On a compact Lie group acting on a manifold, Ann. of Math., (2) 65 (1957), 447-455 and (2) 66 (1957), p. 589.

8. - Conference on compact transformation groups, Springer Verlag, (1969).

Received February 20, 1970. This work was partially supported by the National Science Foundation under Grant No. GP-6167.

UNIVERSITY OF MARYLAND 


\section{PACIFIC JOURNAL OF MATHEMATICS}

\section{EDITORS}

\author{
H. SAMELSON \\ Stanford University \\ Stanford, California 94305 \\ C. R. HовBY \\ University of Washington \\ Seattle, Washington 98105
}

J. DUGUndis

Department of Mathematics

University of Southern California

Los Angeles, California 90007

RICHARD ARENS

University of California

Los Angeles, California 90024

\section{ASSOCIATE EDITORS}
E. F. BeCKenbaCH
B. H. NeUmanN
F. WOLF
K. YoshidA

\section{SUPPORTING INSTITUTIONS}

\author{
UNIVERSITY OF BRITISH COLUMBIA \\ CALIFORNIA INSTITUTE OF TECHNOLOGY \\ UNIVERSITY OF CALIFORNIA \\ MONTANA STATE UNIVERSITY \\ UNIVERSITY OF NEVADA \\ NEW MEXICO STATE UNIVERSITY \\ OREGON STATE UNIVERSITY \\ UNIVERSITY OF OREGON \\ OSAKA UNIVERSITY \\ UNIVERSITY OF SOUTHERN CALIFORNIA
}

\author{
STANFORD UNIVERSITY \\ UNIVERSITY OF TOKYO \\ UNIVERSITY OF UTAH \\ WASHINGTON STATE UNIVERSITY \\ UNIVERSITY OF WASHINGTON

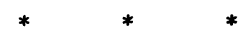 \\ AMERICAN MATHEMATICAL SOCIETY \\ CHEVRON RESEARCH CORPORATION \\ NAVAL WEAPONS CENTER
}

The Supporting Institutions listed above contribute to the cost of publication of this Journal, but they are not owners or publishers and have no responsibility for its content or policies.

Mathematical papers intended for publication in the Pacific Journal of Mathematics should be in typed form or offset-reproduced, (not dittoed), double spaced with large margins. Underline Greek letters in red, German in green, and script in blue. The first paragraph or two must be capable of being used separately as a synopsis of the entire paper. The editorial "we" must not be used in the synopsis, and items of the bibliography should not be cited there unless absolutely necessary, in which case they must be identified by author and Journal, rather than by item number. Manuscripts, in duplicate if possible, may be sent to any one of the four editors. Please classify according to the scheme of Math. Rev. Index to Vol. 39. All other communications to the editors should be addressed to the managing editor, Richard Arens, University of California, Los Angeles, California, 90024.

50 reprints are provided free for each article; additional copies may be obtained at cost in multiples of 50 .

The Pacific Journal of Mathematics is published monthly. Effective with Volume 16 the price per volume (3 numbers) is $\$ 8.00$; single issues, $\$ 3.00$. Special price for current issues to individual faculty members of supporting institutions and to individual members of the American Mathematical Society: $\$ 4.00$ per volume; single issues $\$ 1.50$. Back numbers are available.

Subscriptions, orders for back numbers, and changes of address should be sent to Pacific Journal of Mathematics, 103 Highland Boulevard, Berkeley, California, 94708.

PUBLISHED BY PACIFIC JOURNAL OF MATHEMATICS, A NON-PROFIT CORPORATION

Printed at Kokusai Bunken Insatsusha (International Academic Printing Co., Ltd.), 7-17, Fujimi 2-chome, Chiyoda-ku, Tokyo, Japan. 


\section{Pacific Journal of Mathematics}

Vol. 38, No. $2 \quad$ April, 1971

Richard Davis Anderson and Thomas Ashland Chapman, Extending

homeomorphisms to Hilbert cube manifolds .................. 281

Nguyen Huu Anh, Restriction of the principal series of $\operatorname{SL}(n, \mathbf{C})$ to some

reductive subgroups................................ 295

David W. Boyd, Indices for the Orlicz spaces . . . . . . . . . . . . 315

William Garfield Bridges, The polynomial of a non-regular digraph ...... 325

Billie Chandler Carlson, Robert K. Meany and Stuart Alan Nelson, Mixed

arithmetic and geometric means........................ 343

H. A. Çelik, Commutative associative rings and anti-flexible rings ...... 351

Hsin Chu, On the structure of almost periodic transformation groups ...... 359

David Allyn Drake, The translation groups of n-uniform translation

Hjelmslev planes ................................ 365

Michael Benton Freeman, The polynomial hull of a thin two-manifold . . . . 377

Anthony Alfred Gioia and Donald Goldsmith, Convolutions of arithmetic

functions over cohesive basic sequences .................... 391

Leslie C. Glaser, A proof of the most general polyhedral Schoenflies

conjecture possible ................................

Thomas Lee Hayden and Ted Joe Suffridge, Biholomorphic maps in Hilbert

space have a fixed point ................................ 419

Roger Alan Horn, Schlicht mappings and infinitely divisible kernels ...... 423

Norman Ray Howes, On completeness ...................... 431

Hideo Imai, Sario potentials on Riemannian spaces................ 441

A. A. Iskander, Subalgebra systems of powers of partial universal

algebras.

Barry E. Johnson, Norms of derivations of $\mathscr{L}(\mathrm{X})$.

David Clifford Kay and Eugene W. Womble, Axiomatic convexity theory and relationships between the Carathéodory, Helly, and Radon numbers

Constantine G. Lascarides, A study of certain sequence spaces of Maddox

and a generalization of a theorem of Iyer .............

C. N. Linden, On Blaschke products of restricted growth .

John S. Lowndes, Some triple integral equations ................. 515

Declan McCartan, Bicontinuous preordered topological spaces ......... 523

S. Moedomo and J. Jerry Uhl, Jr., Radon-Nikodým theorems for the Bochner and Pettis integrals ...

Calvin Cooper Moore and Joseph Albert Wolf, Totally real representations

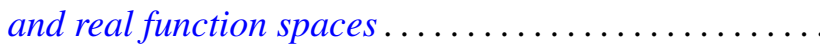

Reese Trego Prosser, A form of the moment problem for Lie groups. ... 